\title{
Amplitude independent versus amplitude dependent muscle activity detection algorithms: a comparative study
}

\author{
Husamuldeen K. Hameed ${ }^{1}$, W. Z. Wan Hasan ${ }^{2}$, Suhaidi Shafie ${ }^{3}$, Siti Anom Ahmad ${ }^{4}$ Haslina Jaafar ${ }^{5}$ \\ $1,2,3,4,5$ Department of Electrical and Electronic Engineering, Faculty of Engineering, Universiti Putra Malaysia, Malaysia \\ ${ }^{2,3}$ Institute of Advanced Technology (ITMA), Universiti Putra Malaysia (UPM), Malaysia
}

\begin{tabular}{l} 
Article Info \\
\hline Article history: \\
Received Mar 25, 2019 \\
Revised May 27, 2019 \\
Accepted Jun 11, 2019 \\
\hline
\end{tabular}

Keywords:

Amplitude independent False alarms

sEMG

Surface electromyography

Weak muscle activity detection

\begin{abstract}
The amplitude dependent muscle activity detection algorithms of the surface electromyography (sEMG) signals are very sensitive to the changes in the background noise levels and the performance of these amplitude-based methods is highly deteriorated when the Signal to Noise ratio (SNR) of the sEMG signal is low. sEMG signals of deep and small muscles as well as sEMG signals recorded from patients that have neuromuscular diseases may not meet this SNR requirement which motivates the need for amplitude independent algorithms that can detect weak muscle activities. Moreover, the sEMG signal amplitude is not constant during the recording time due to the variation in the characteristics of the electrode-skin interface and due to the changes in the ground reference level. Therefore, the performance of the muscle activity detection algorithms should not be affected by the involuntary amplitude variations of the sEMG signal in order to achieve reliable control of robotic devices intended for disabled people. To accentuate the importance of the amplitude independent muscle activity detection methods over the amplitude dependent detection methods, a comparative study has been conducted in this paper between the performance of an amplitude independent muscle activity detection algorithm (FLA-MSE algorithm) and three amplitude dependent algorithms with respect to the detection capability of weak muscle activities and with respect to the immunity against false alarms. The results have showed that the performance of the amplitude independent algorithm outperformed the performance of the amplitude dependent algorithms for detecting weak muscle activities and for robustness against false alarms.
\end{abstract}

Copyright () 2020 Institute of Advanced Engineering and Science. All rights reserved.

Corresponding Author:

Wan Zuha Wan Hasan,

Department of Electrical and Electronic Engineering,

Faculty of Engineering,

Universiti Putra Malaysia, Malaysia.

Email: wanzuha@upm.edu.my

\section{INTRODUCTION}

Because of the stochastic characteristic of sEMG signals, muscle activity detection is a challenging task, especially in weak sEMG signals due to the gradual increase of amplitude and frequency, artefacts noise, spurious background spikes, and random variations in the background noise (usually introduced by electrode movement over the skin) [1]. Many algorithms have been developed in the literature to detect muscle activities from the sEMG signal. The conventional algorithms compare the amplitude parametrs such as the envelope, the average rectified value, the root mean square value of the signal with a predefined threshold value, this threshold must be determined according to the amplitude of the measured signal during no activity period and must be modified whenever the amplitude changes due to many factors. The threshold is user dependent and is cumbersome to optimize with regards to both the detection bias and the false alarm 
probability, where high threshold levels will usually lead to delayed or even missed onset detection; a relatively low threshold level results in early onset detection but also introduces more false alarms [2, 3]. These methods are very popular because of easy implementation, but achieve satisfactory results only when the SNR of the signal is sufficiently high.

Various methods for muscle activity onset detection have been proposed, in which the majority of the used parameters are related to the amplitude of the sEMG signal. For example, wavelet template matching $[4,5]$ was proposed for sEMG muscle activity onset detection by comparing the shape of the surface motor unit action potentials with the appropriate wavelet functions, where good performance can be achieved only when the shape of surface action potentials matches well with the selected wavelet template. However, for experimental sEMG signals, such ideal condition cannot always be guaranteed which may result in a less precise onset detection of muscle activity. Moreover, this detection approach requires large computational power, mainly due to the calculation of the continuous wavelet transform. Qi Xu et al. [2] have developed a detection algorithm based on the maximum likelihood method improved by an adaptive threshold, but the performance of these statistical methods mainly depends on the correct estimation of a priori information of the sEMG signal. Moreover, in statistical detection methods, cumulative sum method requires information about the probability density function which is not available in most real-life problems. Since the firing of action potentials increase the signal amplitude of sEMG signal, the Teager-Kaiser Energy (TKE) operator [6-9] was proposed to highlight this increase and to achieve good performance for the detection of muscle activities especially for sEMG signals that have low SNR. However, the detection methods based on TKE operator was mainly limited to the background noise with Gaussian distribution and they are very sensitive to the spurious background spikes. The authors in [10] have developed a statistical method based on unsupervised learning to model the sEMG signal distribution in the frequency domain; however, this method require intensive computational efforts to process the signal. Vaisman et al. [11], have developed a muscle activity onset detection method based on singular spectrum analysis, but this method need computer to implement the algorithm due to high computational power needed to calculate the expectation- maximization clustering. Furthermore, this method assumes that sEMG signal must have precontraction, contraction and post-contractions parts. Xu and Ping [12] have developed a muscle activity detection algorithm against spurious background spikes by using sample entropy analysis of the sEMG signal, but this method needs high computation efforts and therefore it is difficult to be implemented in real time. Jie and Qiuping [13] have developed a detection algorithm by employing the integrated profile of the sEMG signal in the presence of the spurious background spikes for the spinal cord injury patients, this method depends on the changes in the amplitude of the sEMG signal to report the muscle activities. Severini et al. [14] have improved the Bonato double threshold detection method [15] by making the decision threshold value adaptive to the changes in the sEMG signal to noise ratio. Most of the aforementioned methods require either prior knowledge about the EMG generating model or intensive calculations making them impractical for real-time applications because many of them are not intended to control robotic devices but for other applications like neurological diagnosis or sports medicine. Moreover, statistically optimal decision, frequency domain analysis, and machine learning detection methods need heavy computational requirements that would impose burdens on real time implementation especially for embedded systems, while real-time system demands fast algorithms having low computational complexity.

In order to emphasize the importance of employing amplitude independent muscle activity detection algorithms to control hand robotic devices used for disabled people, a comparison has been made in this paper between the performance of the amplitude independent muscle activity detection algorithm presented in $[30,31]$ (FLA-MSE algorithm) and three amplitude dependent algorithms (Rectified and filtered sEMG, Teager Kaiser Energy Operator, Integrated Profile). All the four algorithms have low computational efforts and can be implemented in real time. The comparison has been conducted with respect to the ability of detecting weak muscle activities and with respect to the immunity against false alarms caused by involuntary amplitude variations in the sEMG signals (false alarms cause unintended movements of the robotic devices).

\section{THE FLA-MSE ALGORITHM}

The FLA-MSE algoritm is an amplitude independent muscle activity detection algorithm developed by Husamuldeen et al. [30] and it was successfully employed to control the movement of a soft robotic glove. The algorithm employs the First Lag Autocorrelation (FLA) and the Modified Sample Entropy (MSE) to detect weak muscle activities from sEMG signals. The algorithm is also immune against spurious background spikes that may contaminate the sEMG signal and degrade the performance of the amplitude dependent muscle activity detection methods. Moreover, the algorithm is computation efficient to enable real time implementation and can distinguish between two types of muscle activities "hand close" and "hand open" by using single sEMG channel without the need to apply the classification algorithms. 
The distinguishing procedure of the FLA-MSE algorithm is illustrated in Figure 1, Figure 2 shows a sample of the performance of the algorithm to detect and classify two muscle activities "hand close" and "hand open" from real sEMG signal obtained from the forearm muscles of a healthy subject.

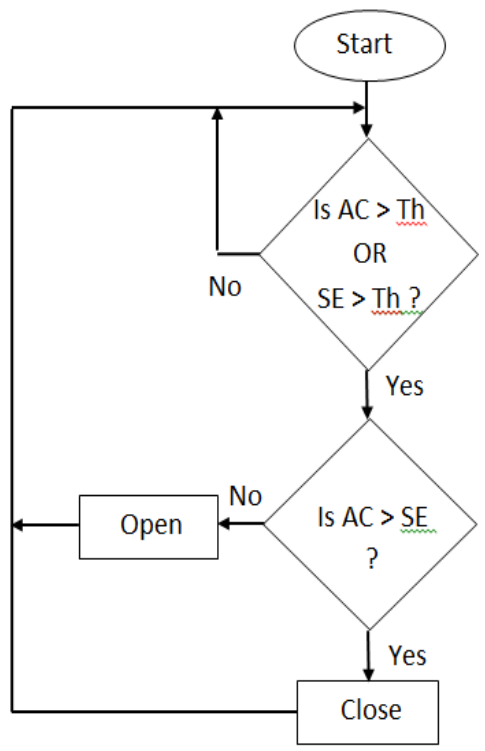

Figure 1. Muscle activity distinguishing flow chart

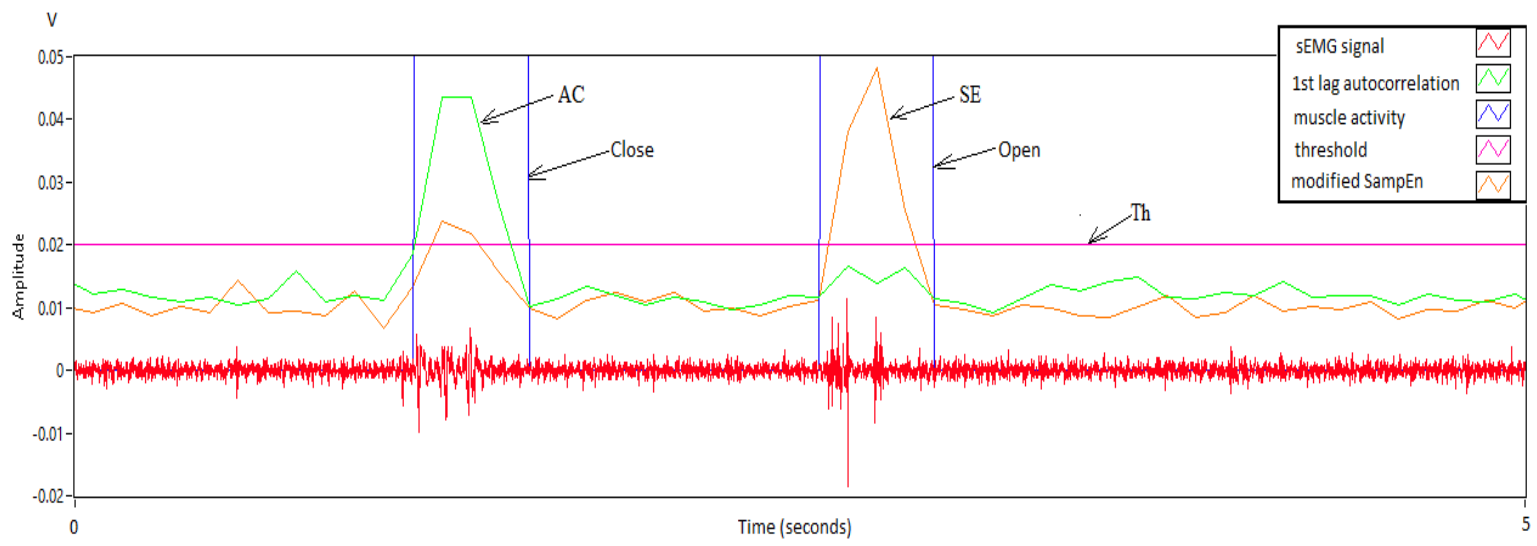

Figure 2. sEMG signal with one "close" and one "open" activity

\section{PERFORMANCE COMPARISON BETWEEN THE FLA-MSE ALGORITHM AND THREE AMPLITUDE DEPENDANT MUSCLE ACTIVITY DETECTION ALGORITHMS}

To evaluate the performance of the amplitude independent muscle activity detection method against amplitude dependent detection methods, a comparison has been conducted between the performance of the amplitude independent FLA-MSE algorithm and three amplitude dependent detection methods. The first method is the classical detection method which compares the rectified and filtered sEMG signal with a predefined threshold, where this method was used in most of the practical implemented hand robotic devices [16-25]. The second method is the Teager Kaiser Energy Operator (TKE) [7-10,26] and the third method is the Integrated Profile (IP) $[13,27]$ of the sEMG signal. These three methods were chosen because they can be implemented in real time and have low computational efforts. The comparison was done with respect to the ability of detecting weak muscle activities and with respect to the immunity against false alarms, where these two parameters are the most important parameters that a muscle activity detection algorithm must have for reliable control of hand robotic devices intended for disabled people. 
For the first method, each sEMG signal segment was full wave rectified and filtered by a 4th order Butterworth IIR low pass filter with a cutoff frequency of $10 \mathrm{~Hz}$ as was used in [17] and as suggested by International Society of Electrophysiology and Kinesiology (ISEK) guidelines [28]. The rectified and filtered sEMG signal was compared with a threshold value which is three times standard deviation of the sEMG signal during no activity period as suggested in [29], a muscle activity is reported when the signal exceeds the threshold value. For the second method, the TKE operator was computed for each sEMG segment and the result was compared with a threshold of eight times standard deviation of sEMG signal during no activity period as suggested in [9]. For the third method, the integrated profile algorithm [13] was applied for each sEMG segment.

\subsection{Comparison with Respect to the Detection Capability}

The hardware setup shown in Figure 3 was used to conduct a comparison among the four algorithms (FLA-MSE algorithm, rectified and filtered, TKE, IP) according to the flow diagram illustrated in Figure 4 to test the ability of detecting weak muscle activities. A healthy subject wearing soft glove on his left hand has generated ten weak 'close' muscle activities from his Flexor Carpi Ulnaris (FCU) muscle as suggested in [30]. The amplitude independent FLA-MSE algorithm has managed to detect all the ten muscle activities as illustrated in Figure 5, while the IP method has detected six muscle activities, rectified and filtered method and TKE method both have detected four muscle activities. Figure 6 shows a zoomed sEMG signal of Figure 5.

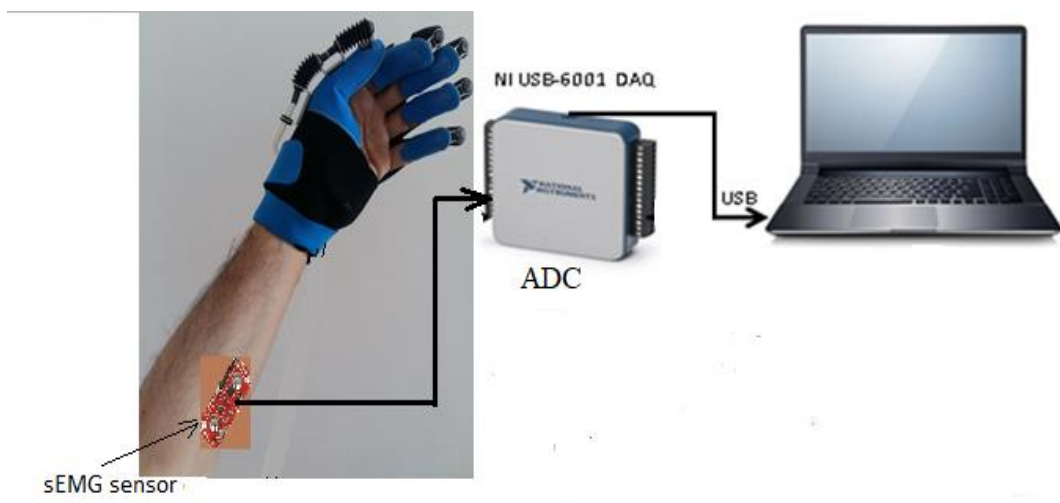

Figure 3. The hardware setup used in the experiments

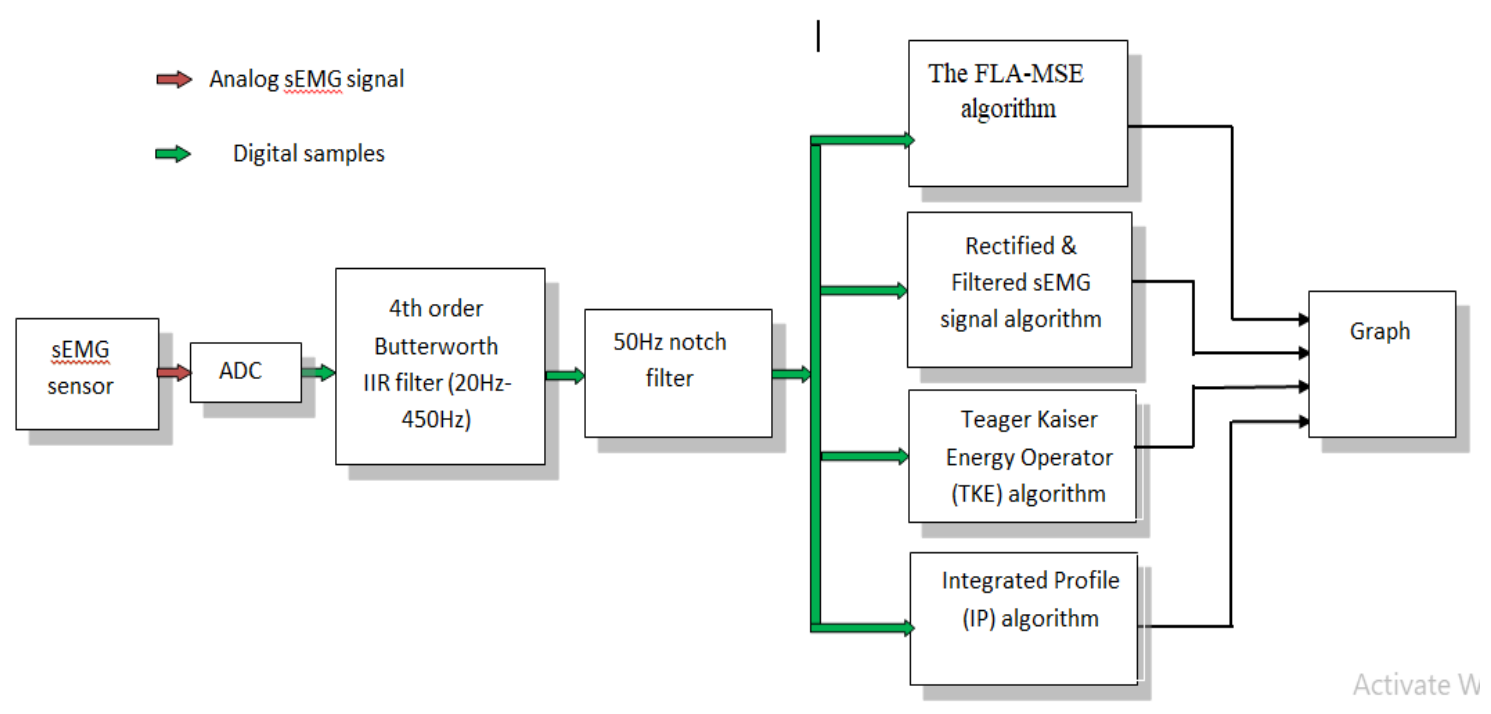

Figure 4. Flow Diagram used for the comparison among the four algorithms 


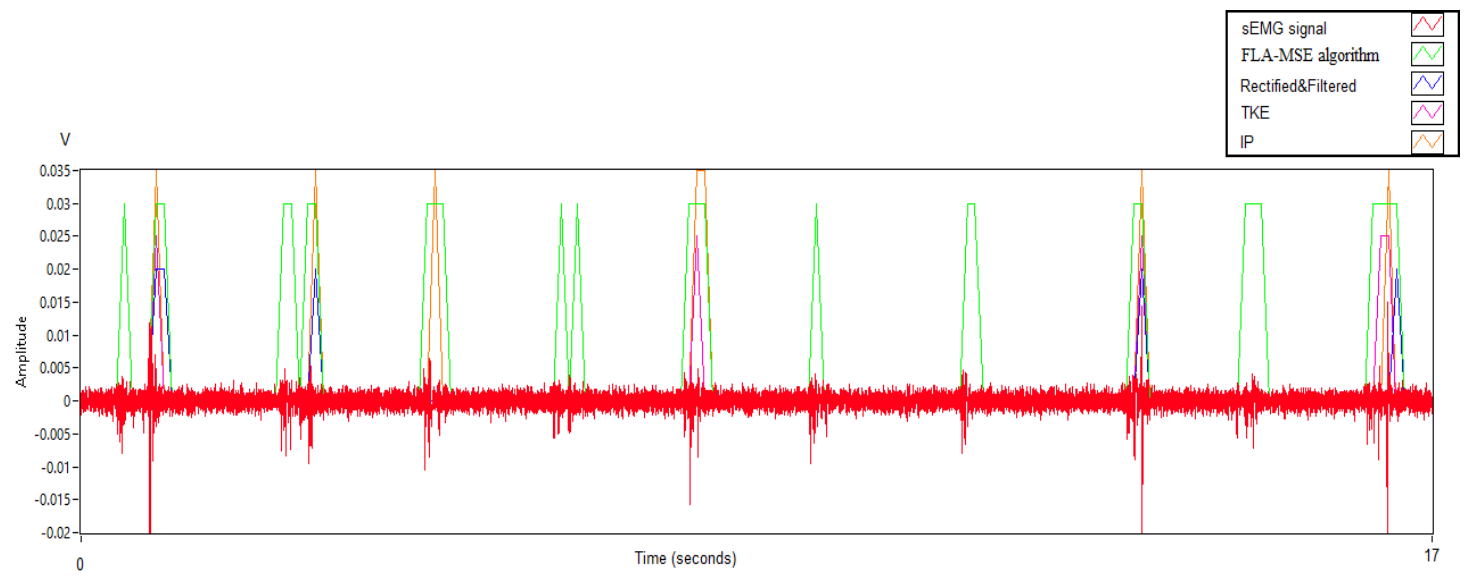

Figure 5. sEMG signal with ten muscle activities. Comparison among the four algorithms with respect to the ability of detecting weak muscle activities

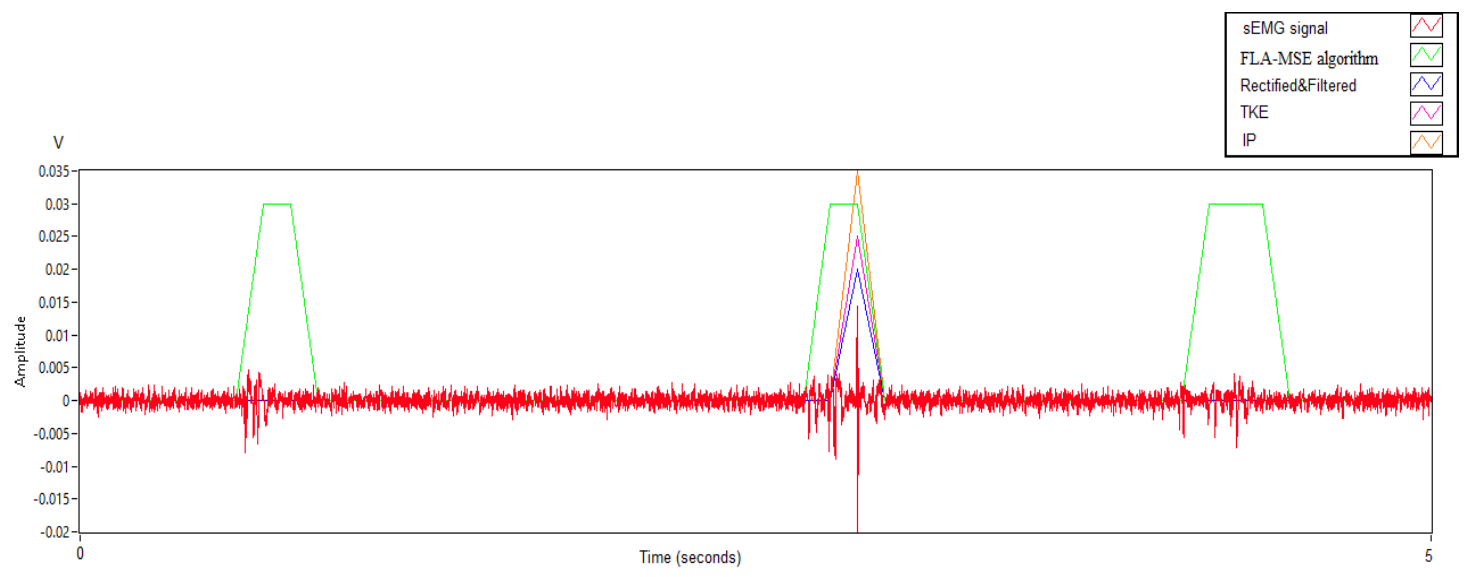

Figure 6. sEMG signal with three muscle activities. Zoomed sEMG signal of Figure 5

To test the reliability and robustness of the detection capability of the amplitude independent FLA-MSE algorithm with respect to the inter-experimental variations, three rounds were conducted in different times with new doff and don of the sEMG channel for each round. Each round was including a generation of 25 weak 'close' muscle activities by a healthy subject wearing soft glove on his left hand. The sEMG channel was located on the Flexor Carpi Ulnaris (FCU) muscle of the forearm. As shown in table 1 and Figure 7, the FLA-MSE algorithm has succeeded to detect all the muscle activities. There was no difference of the outcomes for experiments conducted on different times, indicating that there was no impact of inter-experimental variations on the efficacy of the amplitude independent method to detect weak muscle activities. In contrast, the amplitude dependent methods have given different performance for each round because the amplitude of sEMG signals was different in each round due to the reinstalling and installing of the sEMG channel and consequently changing in the electrode-skin interface characteristics.

Table 1. Numbers of the Muscle Activity Detections for the Three Rounds

\begin{tabular}{|c|c|c|c|c|}
\hline \multirow[b]{2}{*}{ Round No. } & \multicolumn{4}{|c|}{ No. of true detected muscle activities out of 25 activities } \\
\hline & FLA-MSE algorithm & $\begin{array}{l}\text { Rectified and filtered } \\
\text { sEMG }\end{array}$ & $\begin{array}{c}\text { Teager Kaiser } \\
\text { Energy operator } \\
\text { (TKE) }\end{array}$ & Integrated Profile (IP) \\
\hline $1 \mathrm{st}$ & 25 & 2 & 10 & 4 \\
\hline 2nd & 25 & 1 & 1 & 1 \\
\hline $3 \mathrm{rd}$ & 25 & 7 & 7 & 7 \\
\hline
\end{tabular}




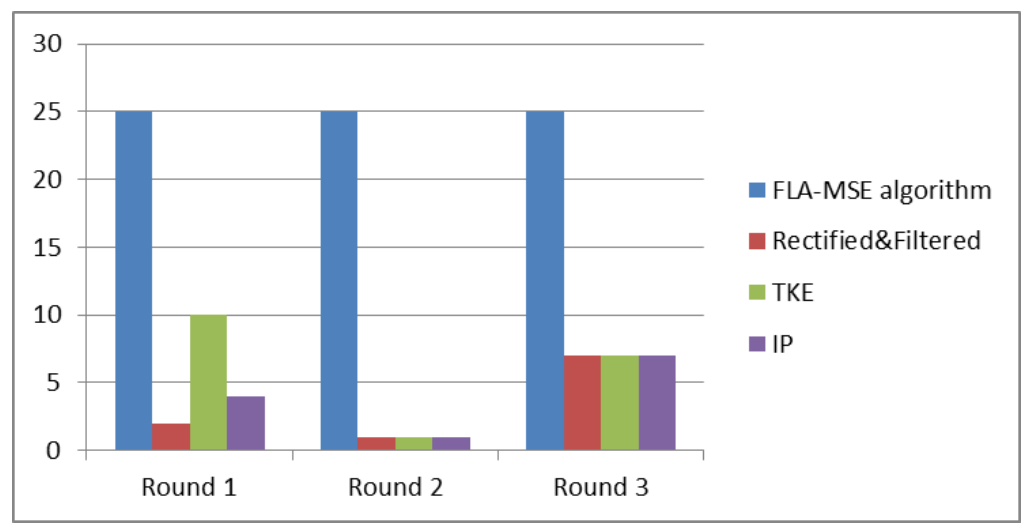

Figure 7. Numbers of true detected muscle activities by the amplitude independent FLA-MSE algorithm compared to the other amplitude dependent algorithms

Figures 8, 9, and 10 with 5 seconds window span show samples of the muscle activity detections for the three rounds, where round 1 was with noisy sEMG signal and round 2 was with very weak muscle activities. The difference among the three rounds with respect to the sEMG signal was due to the changing in the electrodes contact with the skin caused by don and doff of the sEMG channel.

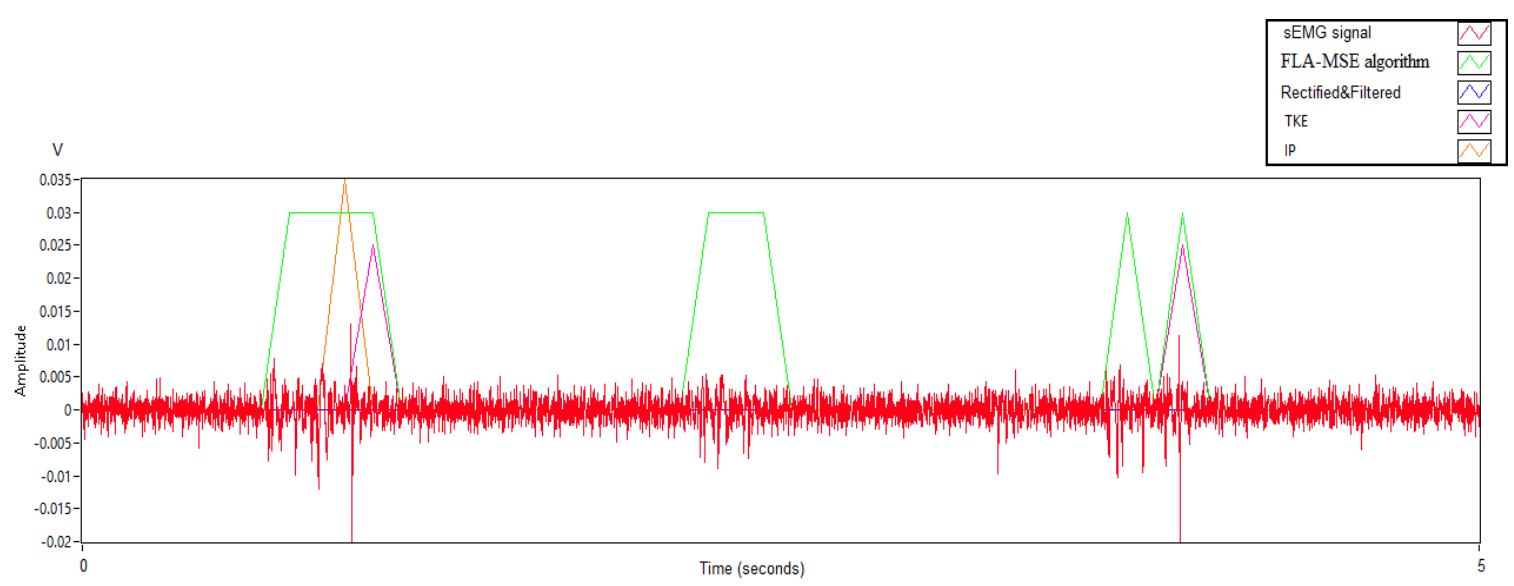

Figure 8. sEMG signal with three muscle activities from the FCU muscle for round 1

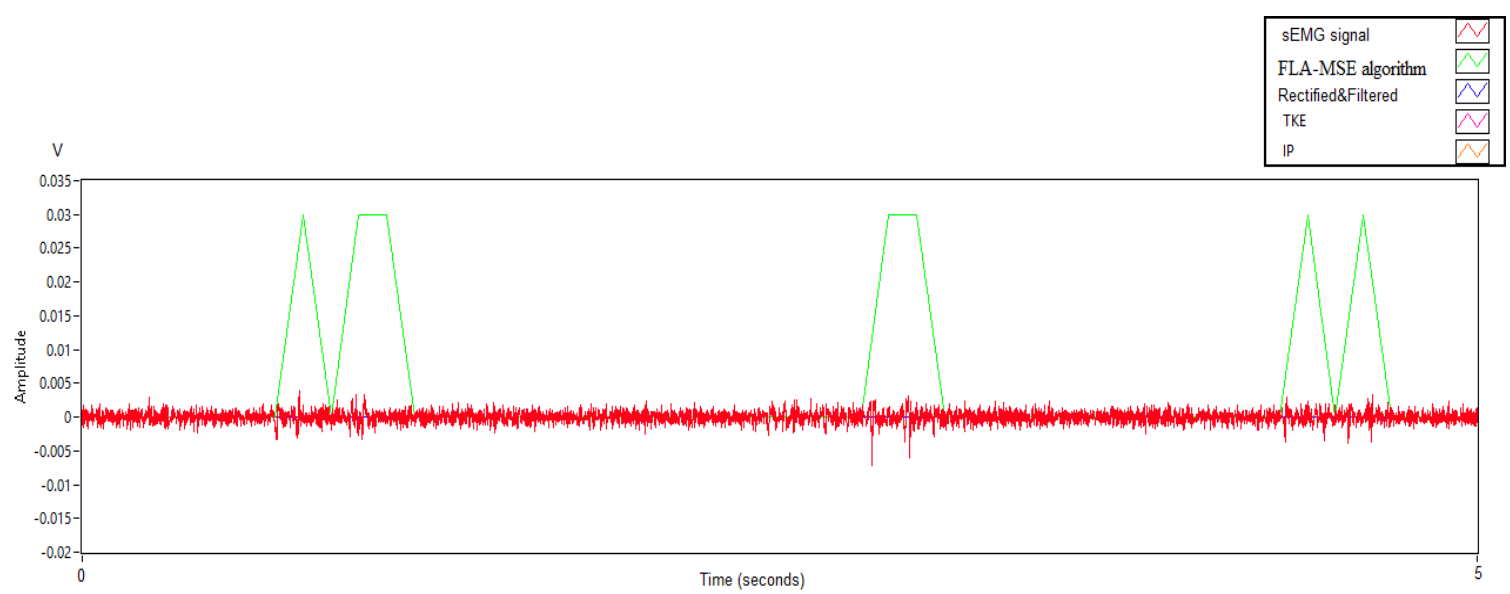

Figure 9. sEMG signal with three muscle activities from the FCU muscle for round 2 


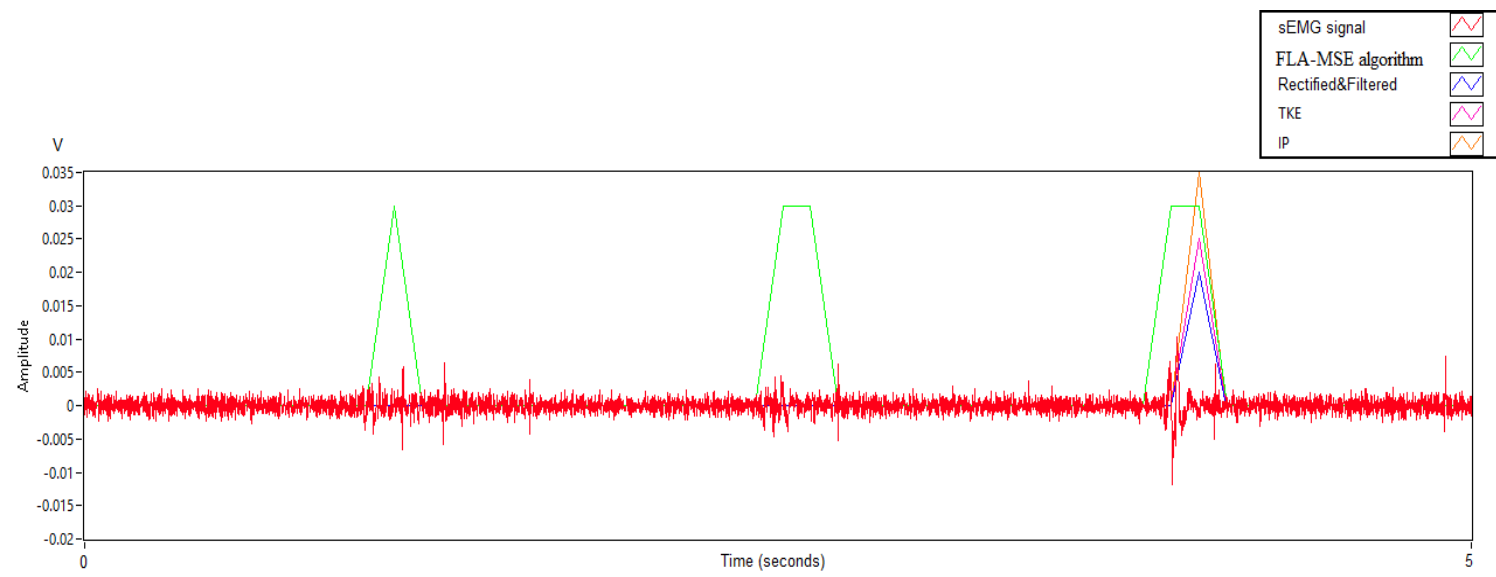

Figure 10. sEMG signal with three muscle activities from the FCU muscle for round 3

To compare the performance of the four algorithms for detecting (not distinguishing) different types of muscle activities like "Close" and "Open" from the Flexor Carpi Ulnaris (FCU) muscle, twenty "Close" activities then twenty "Open" activities were generated by a healthy subject wearing soft glove on his left hand. The numbers of the detected "Close" and "Open" muscle activities by the four algorithms are shown in Table 2 and Figure 11.

Table 2. Numbers of the Detected "Open" and "Close" Muscle Activities by the Four Algorithms

\begin{tabular}{ccccc}
\hline $\begin{array}{c}\text { Muscle activity } \\
\text { from FCU muscle }\end{array}$ & FLA-MSE algorithm & $\begin{array}{c}\text { No. of the detected muscle activities out of } 20 \text { activities } \\
\text { Rectified and filtered } \\
\text { TEMG }\end{array}$ & $\begin{array}{c}\text { Teager Kaiser } \\
\text { Energy operator } \\
\text { (TKE) }\end{array}$ & Integrated Profile (IP) \\
\hline "Close" & 20 & 8 & 12 & 9 \\
"Open" & 20 & 6 & 20 & 8 \\
\hline
\end{tabular}

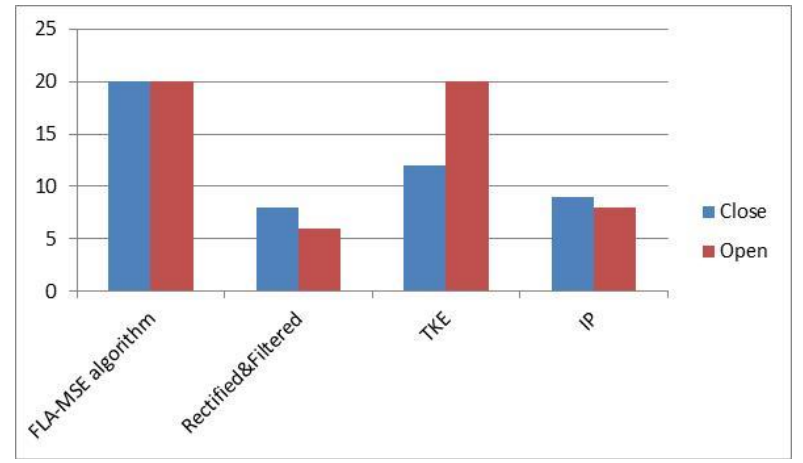

Figure 11. Detected "Open" and "Close" muscle activities by the four algorithms

The amplitude independent FLA-MSE algorithm has succeeded to detect all the "Close" and "Open" activities which is not the case for the other three amplitude dependent algorithms (keeping in mind that just the FLA-MSE algorithm can distinguish between "Close" and "Open activities). With respect to the "Close" activities, the other three algorithms have failed to detect many of them due to the low SNR of this activity. With respect to the "Open" activities, the TKE algorithm has managed to detect all of them due to the good SNR of this activity from the FCU muscle, whereas the "Rectified \& Filtered" and the "Integrated Profile" algorithms have failed to detect many of them due to the nature of this activity which is composed of sharp pulses. Figure 12 and Figure 13 show samples of the sEMG signal that was used to do the comparison, where sEMG signal with four detected "Close" activities and four detected "Open" activity are appeared in a 5 second window span. 


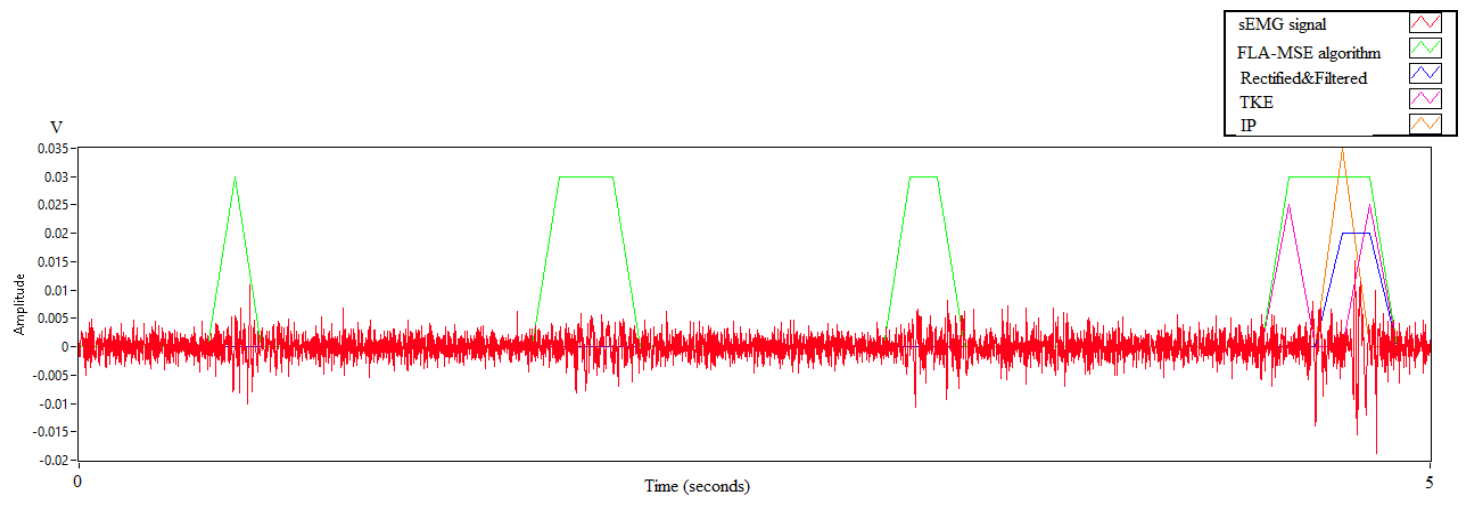

Figure 12. sEMG signal with four "Close" muscle activities from the FCU muscle

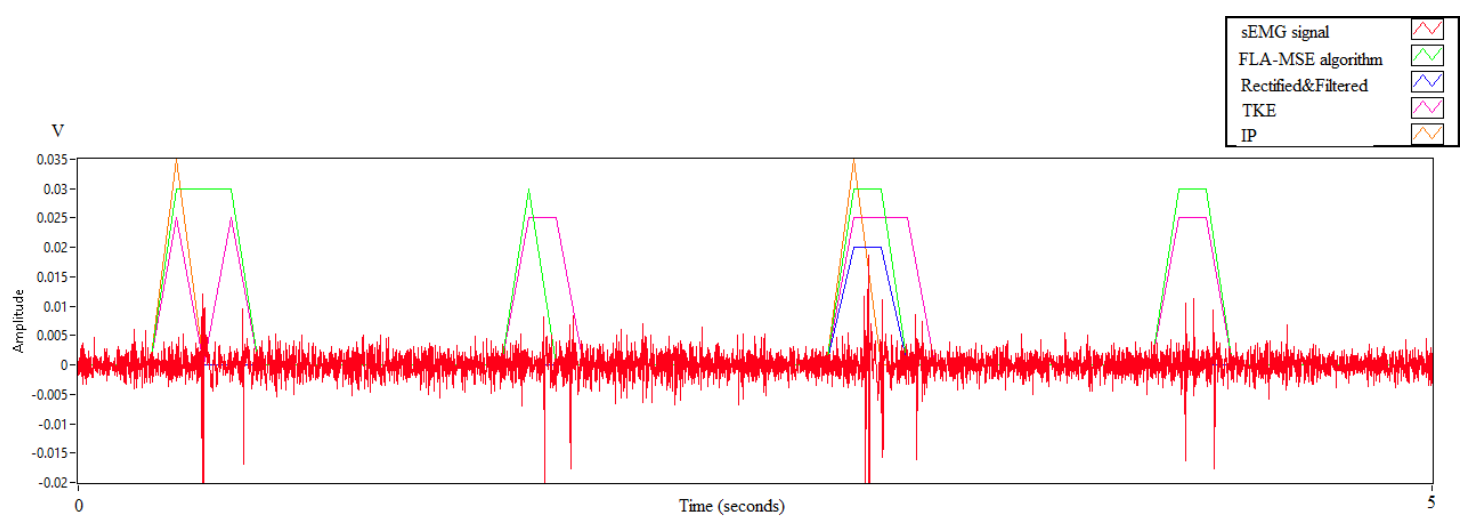

Figure 13. sEMG signal with four "Open" muscle activities from the FCU muscle

\subsection{Comparison with respect to the immunity against false alarms}

To compare the robustness of the four algorithms against false alarms caused by amplitude variation of the sEMG signal, an Additive White Gaussian Noise (AWGN) with a standard deviation of $5 \mathrm{mV}$ was added to a real sEMG signal. A real sEMG signal without muscle activities was obtained from the Flexor Carpi Ulnaris muscle of a healthy subject and the AWGN was added five times to the signal to simulate an amplitude variation for the sEMG signal. As shown in Figure 14, the amplitude independent FLA-MSE algorithm was immune against amplitude variation and there is no false alarm, while the other algorithms have produced false alarms for each amplitude variation.

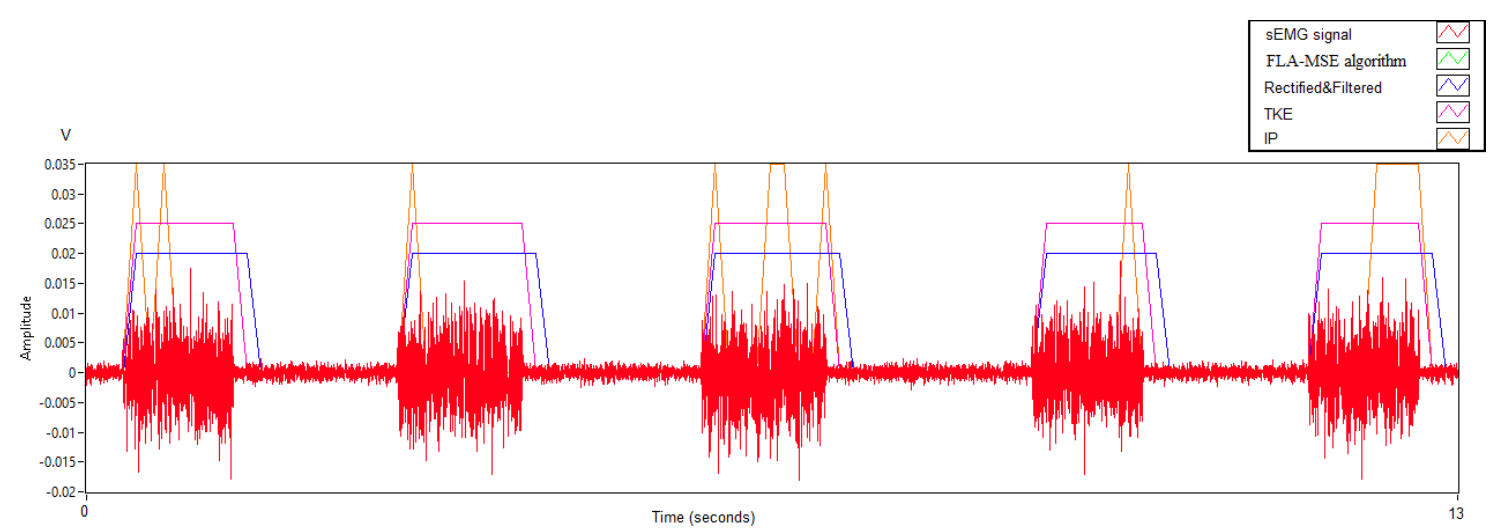

Figure 14. sEMG signal without muscle activities and with five AWGN. Comparison among the four algorithms with respect to immunity against false alarms 


\section{CONCLUSIONS}

The sEMG signal amplitude characteristics differ from one person to another and from time to time according to many factors like the electrode location, sweat, fatigue, blood circulation, the electrical impedance of the skin, the thickness of adipose tissue, and body temperature. Moreover, it is found in previous studies that motor unit over activity in stroke patients are sometimes present as a background signal that may contaminate the voluntary sEMG signal of the impaired muscles and make it difficult to use the conventional amplitude dependant methods for detecting muscle activities of neurological injury patients. In this paper, a comparison has been conducted between the performance of the amplitude independent FLAMSE algorithm presented in [30] and three amplitude dependent muscle activity detection algorithms with respect to the ability of detecting weak muscle activities and with respect to the immunity against false alarms caused by involuntary amplitude variations. The sEMG signal was obtained by using single channel from the forearm muscles of a healthy subject wearing soft robotic glove in his left hand. First of all, ten weak musle activities were generated from the Flexor Carpi Ulnaris (FCU) muscle and a comparison among the four algorithms was done with respect to the detection capability, where the amplitude independent algorithm has detected all the ten activities. To test the reliability and robustness of the detection capability of the amplitude independent algorithm with respect to the inter-experimental variations, three rounds were conducted in different times with new doff and don of the sEMG channel for each round, 25 muscle activities were generated for each round. The results have revealed that the detection capability of the amplitude independent algorithm outperformed the amplitude dependent algorithms with respect to the detection of weak muscle activities. To compare the performance of the four algorithms for detecting different types of muscle activities, 20 hand "Close" activities and 20 hand "Open" activities were used in the comparison. The FLA-MSE algorithm has succeeded to detect all the muscle activities which is not the case for the other algorithms. Finally, a comparison has been conducted among the four algorithms to test the robustness against false alarms caused by involuntary amplitude variations. The result has confirmed the robustness of the amplitude independent method against false alarms in contrary to the amplitude dependent methods. Employing amplitude independent muscle activity detection methods for controlling hand robotic devices intended for disabled people makes these devices more reliable to be used in daily basis.

\section{ACKNOWLEDGMENT}

The author would like to thank Universiti Putra Malaysia for supporting this research work under supervision and Ministry of Education for funding this study under the MOE-FRGS scheme (03-01-17-1893FR).

\section{REFERENCES}

[1] J. Drapala, et al., "Two stage EMG onset detection method,” Arch. Control Sci., vol. 22, no. 4, pp. 427-440, 2012.

[2] Q. Xu, et al., "An adaptive algorithm for the determination of the onset and offset of muscle contraction by EMG signal processing," IEEE Trans. Neural Syst. Rehabil. Eng., vol. 21, no. 1, pp. 65-73, 2013.

[3] G. Staude, et al., "Onset detection in surface electromyographic signals: A systematic comparison of methods," EURASIP J. Appl. Signal Processing, vol. 2, pp. 67-81, 2001.

[4] R. Andrea Merlo and F. Dario, "A Fast and Reliable Technique for Muscle Activity Detection From Surface EMG Signals," IEEE Trans. Biomed. Eng., vol. 50, no. 3, pp. 319-323, 2003.

[5] G. Vannozzi, et al., "Automatic detection of surface EMG activation timing using a wavelet transform based method," J. Electromyogr. Kinesiol., vol. 20, no. 4, pp. 767-772, 2010.

[6] D. Yang, et al., "Accurate EMG onset detection in pathological, weak and noisy myoelectric signals," Biomed. Signal Process. Control, vol. 33, pp. 306-315, 2017.

[7] A. Kaur, et al., "Adaptive threshold method for peak detection of surface electromyography signal from around shoulder muscles," J. Appl. Stat., vol. 45, no. 4, pp. 714-726, 2018.

[8] X. Li, et al., "Teager-kaiser energy operation of surface EMG improves muscle activity onset detection," Ann. Biomed. Eng., vol. 35, no. 9, pp. 1532-1538, 2007.

[9] S. Solnik, et al., "Teager-Kaiser operator improves the accuracy of EMG onset detection independent of signal-to-noise ratio," Acta Bioeng. Biomech., vol. 10, no. 2, pp. 65-68, 2008.

[10] J. Liu, et al., "Robust muscle activity onset detection using an unsupervised electromyogram learning framework," PLoS One, vol. 10, no. 6, pp. 1-15, 2015.

[11] L. Vaisman, et al., "Application of singular spectrum-based change-point analysis to EMG-onset detection," $J$. Electromyogr. Kinesiol. vol. 20, no. 4, pp. 750-760, 2010.

[12] X. Zhang and P. Zhou, "Sample entropy analysis of surface EMG for improved muscle activity onset detection against spurious background spikes," J. Electromyogr. Kinesiol., vol. 22, no. 6, pp. 901-907, 2012.

[13] J. Liu and Q. Liu, "Use of the integrated profile for voluntary muscle activity detection using EMG signals with spurious background spikes: A study with incomplete spinal cord injury," Biomed. Signal Process. Control, vol. 24, pp. 19-24, 2016. 
[14] G. Severini, et al., "Novel formulation of a double threshold algorithm for the estimation of muscle activation intervals designed for variable SNR environments," J. Electromyogr. Kinesiol., vol. 22, no. 6, pp. 878-885, 2012.

[15] P. Bonato, et al., "A statistical method for the measurement of muscle activation intervals from surface myoelectric signal during gait," IEEE Trans. Biomed. Eng., vol. 45, no. 3, pp. 287-299, 1998.

[16] J. Ngeo, et al., "Control of an Optimal Finger Exoskeleton based on Continuous Joint Angle Estimation from EMG signals," 35th Annual International Conference of the IEEE Engineering in Medicine and Biology Society, Osaka, Japan, pp. 338-341, 2013.

[17] Ahmed M. M. ALmassri, et al. "Self-Calibration Algorithm for an Intelligent Sensor with a Real-Time Approach Based on an Artificial Neural Network" Sensors, pg1-16, 2018.

[18] H. Yap, et al., "A Fabric-Regulated Soft Robotic Glove with User Intent Detection using EMG and RFID for Hand Assistive Application," IEEE International Conference on Robotics and Automation, Stockholm, Sweden, pp. 3537-3542, 2016.

[19] T. Kline, et al. "Control System for Pneumatically Controlled Glove to Assist in Grasp Activities," 9th International Conference on Rehabilitation Robotics, Chicago, IL, USA, pp.78-81, 2005.

[20] M. Delph, et al., "A Soft Robotic Exomusculature Glove with Integrated sEMG Sensing for Hand Rehabilitation," IEEE 13th International Conference on Rehabilitation Robotics, Seattle, WA, USA, pp. 1-7, 2013.

[21] J. Ochoa and D. Kamper, "Development of an actuated cable orthotic glove to provide assistance of finger extension to stroke survivors," Rev. Ing. Biomédica, vol. 3, no. 5, pp. 75-829, 2009.

[22] K. Thielbar, et al., "Benefits of using a voice and EMG-driven actuated glove to support occupational therapy for stroke survivors," IEEE Transactions on Neural Systems and Rehabilitation Engineering, vol. 25, no. 3, pp. 297-306, 2017.

[23] L. Lucas, et al., "An EMG-Controlled Hand Exoskeleton for Natural Pinching," J. Robot. Mechatronics, vol. 16, no. 5, pp. 482-488, 2004.

[24] M. Mulas, et al., "An EMG-controlled exoskeleton for hand rehabilitation," 9th International Conference on Rehabilitation Robotics ICORR, Chicago, IL, USA, pp. 371-374, 2005.

[25] M. Chen, et al., "Interactive rehabilitation robot for hand function training," IEEE International Conference on Rehabilitation Robotics ICORR. Kyoto, Japan, pp. 777-780, 2009.

[26] P. Gentile, et al., "Embedded wearable integrating real-time processing of electromyography signals," Eurosensors 2017 conference, Paris, France, 3-6 September 2017. Proceedings 2017, 1, 600; doi: 10.3390/ proceedings 1040600 .

[27] F. N. Jamaluddin, et al. "Low cost and wearable multichannel surface electromyography data acquisition system architecture" Journal of Engineering Science and Technology, pp 98-106, 2014.

[28] "Standards for Reporting EMG data," Journal of Electromyography and Kinesiology, vol. 9, no. 1, III-IV, February 1999.

[29] P. Hodges et al., "A comparison of computer-based methods for the determination of onset of muscle contraction using electromyography," Electroencephalography and clinical Neurophysiology, vol. 101, pp. 511-519, 1996.

[30] H. Hameed, et al., "Soft robotic glove system controlled with amplitude independent muscle activity detection algorithm by using single sEMG channel," IEEE 5th International Conference on Smart Instrumentation, Measurement and Applications (ICSIMA 2018). Songkhla, Thailand, pp. 1-6, 2108.

\section{BIOGRAPHIES OF AUTHORS}

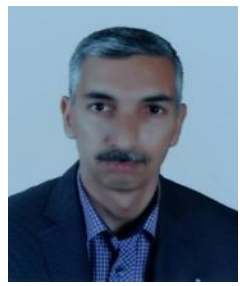

Husamuldeen K. Hameed received the Bachelor and Master Degrees in Electrical and Electronic Engineering from the University of Technology in 1994 and 2002 respectively. He is a lecturer in the High Institute of Telecommunications. He is currently a $\mathrm{PhD}$ student at the department of Electrical and Electronic Engineering in the Universiti Putra Malaysia. His PhD study is about Electromyography controlled hand robotic devices for disabled people. His research interests include Digital Signal Processing, Biomedical, Robotics and Automation.

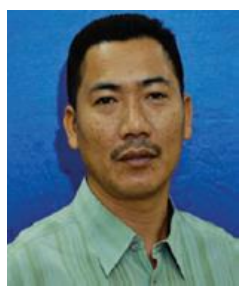

Wan Zuha Wan Hasan is a Senior Lecturer of Electrical and Electronic Engineering, Faculty of ngineering, Universiti Putra Malaysia (UPM). He received the B.Sc. degree in Electronic and Computer from the Universiti Putra Malaysia (UPM) in 1997, and the M.Sc. in Microelectronics from the Universiti Putra Malaysia (UPM) in 2001, and the Ph.D. in Memory Testing from the University Kebangsaan Malaysia (UKM) in 2005. He is a member IEEE member (BEM), IEEE Circuit and System Society (IEICE) and IEEE Electron and Devices (PPAUPM). His areas of specialization are Microelectronics, Sensor Technology, Robotic and Automation, especially in Pressure Sensor for Medical and Robotic and Automation Application, Mobile Robot and automated Machine. 


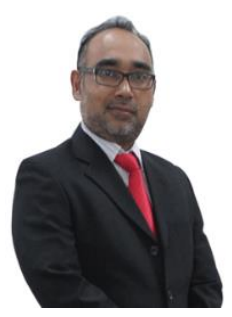

Suhaidi Shafie received the Bachelor of Engineering (Electrical and Electronics) from University of the Ryukyus, Japan in 2000. From 2000 to 2002, he was with ALPS Electric (M) Sdn. Bhd. $\mathrm{He}$ received the Master of Engineering (Electrical and Electronics) from Tokyo University of Agriculture and Technology, and the Doctor of Engineering (Nanovision) from Shizuoka University in 2005 and 2008, respectively. He is an Associate Professor in Universiti Putra Malaysia and the Head of Functional Devices Laboratory. Dr. Suhaidi is working in Mix Signal IC Design and Solar Energy research. His current projects include Ultra Low Power SAR ADC and High Efficiency Dye Sensitized Solar Cell. He is was the chapter chair of IEEE Circuits and Systems Malaysia Chapter and actively involves in IEEE CAS and IMS Malaysia Chapters activities.

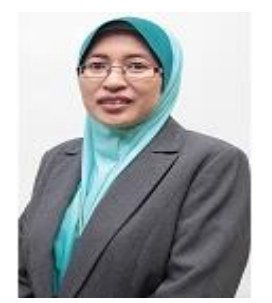

Siti Anom Ahmad is an Associate Professor at the Department of Electrical and Electronic Engineering, Faculty of Engineering, Universiti Putra Malaysia. She received her BEng in Electronic/ Computer from Universiti Putra Malaysia in 1999. Dr. Siti Anom received a Ph.D. in Electronics in 2009 and MSc in Microelectronics System Design in 2004 from the University of Southampton, UK. Her research interests are biomedical engineering, signal processing and intelligent control system. She has published more than 100 journal articles and proceedings in her field.

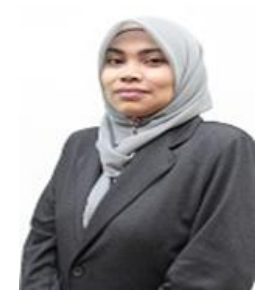

Haslina Jaafar is a senior lecturer at the Department of Electrical and Electronic Engineering, Faculty of Engineering, University of Putra Malaysia (UPM). She received her BE in Electrical and Electronic Engineering and Systems and MS degree in microelectronics from National University of Malaysia (UKM) in 1999 and 2001, respectively, and her $\mathrm{PhD}$ degree in Microelectro-mechanical systems from the University Science Malaysia (USM) in 2014. Her current research interests include MEMS, flexible electronics and nanotechnology. She is a senior member of IEEE, active members of Electron Devices Society (EDS) and Circuits and Systems Society (CAS). 